\section{Año 2019-2020. urtea} N. ${ }^{\circ}$ 31-32. zk.

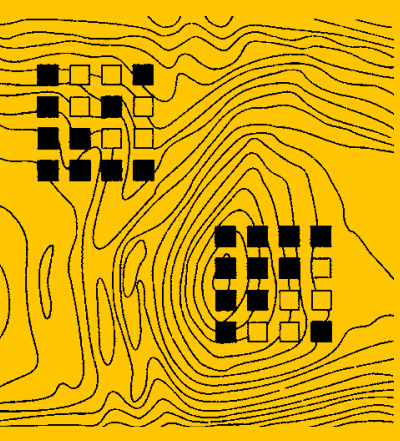

\section{TRABAJOS DE ARQUEOLOGÍA NAVARRA}

SEPARATA

\title{
Proyecto de intervención en la cueva de Ostolo (Arantza, Navarra). Campañas de 2017-2020
}




\section{Proyecto de intervención en la cueva de Ostolo (Arantza, Navarra). Campañas de 2017-2020}

Esku-hartze arkeologikoa Ostolo koban (Arantza, Nafarroa). 2017-2020 ekinaldiak Archaeological research in Ostolo cave (Arantza, Navarre). 2017-2020 field seasons

Jesús Tapia Sagarna

Aranzadi Zientzia Elkartea

jtapia@aranzadi.eus

DOI: https://doi.org/10.35462/TAN31-32.10 


\section{RESUMEN}

Ostolo fue descubierta en 2006 y, tras una prospección en 2015, desde 2017 desarrollamos un proyecto de investigación para analizar las condiciones físicas de la cueva y su registro arqueológico. La entrada a Ostolo se realiza a través de una estrecha surgencia, y una vez dentro, los restos se encuentran en superficie sobre una terraza. En los tres conjuntos diferenciados se han hallado restos humanos y ofrendas, destacando un brazal de arquero y un botón de doble perforación en V. La datación disponible concuerda con los materiales y los enterramientos en cueva, durante el Campaniforme. El próximo paso será analizar este comportamiento simbólico y las condiciones de acceso a la cueva.

Palabras clave: Edad del Bronce; cultura campaniforme; cuevas sepulcrales; prehistoria; Navarra.

\section{LABURPENA}

Ostolo 2006an aurkitu zen, eta 2015ean miaketa arkeologiko bat egin ondoren, 2017tik aurrera, proiektu bat martxan jarri dugu kobaren baldintza fisikoak eta ondare arkeologikoa aztertzeko. Ostoloko sarbide bakarra iturri estu bat da, eta barruan, aztarnak terraza baten gainean aurkitzen dira, lurrazalean. Hiru multzo bereiztu ditugu giza hezur eta ostilamendu batzuekin, eta horien artean azpimarratu behar dira arkularibesoko bat eta V-formako zulo bikoitzeko botoi bat. Orain arte lortu dugun datazioa bat dator materialekin eta hilobi-haitzuloen fenomenoarekin, Kanpai-formako Kulturaren garaikoak. Hurrengo pausoa portaera sinbolikoak eta koban sartzeko baldintzak aztertzea izango da.

Gako hitzak: Brontze Aroa; Kanpai-formako Kultura; hilobi-haiztuloak; Historiaurrea; Nafarroa.

\section{ABSTRACT}

Ostolo was discovered in 2006, and after a survey carried out in 2015, we develop a research project since 2017 to analyze the physical conditions of the cave and study its archaeological record. The only entrance to Ostolo is a narrow spring, and inside, archaeological remains appear on surface upon a terrace. In the three sets that we have differentiated human remains and offerings are found, among which a wrist-guard and a V-shape button stand out. The date obtained is consistent with the materials and with the cave burials during the Bell-Beaker horizon. The next step will be to analyze this symbolic behavior and determine the access conditions to the cave in that time.

Keywords: Bronze Age; Bell-Beaker Culture; cave burials; Prehistory; Navarre. 
La cueva de Ostolo (Arantza) fue descubierta a raíz de unas exploraciones espeleológicas realizadas por Iñaki Alzuri, Koldo Sánchez y Nacho del Cura en 2006, quienes topografiaron la cavidad y localizaron en su interior varios restos óseos en superficie. Algunos de estos materiales fueron recogidos y llevados para su consulta a F. Etxeberria, que confirmó que se trataba de restos humanos atribuibles al Calcolítico, y en 2007 entregó los restos al Museo de Navarra acompañados de un informe antropológico.

Sin embargo, este nuevo yacimiento no fue objeto de mayor atención hasta el año 2012, en que, ante la preocupación de los descubridores por la degradación del yacimiento, organizamos una visita para valorar su estado de conservación y su potencial arqueológico.

Como resultado de esa exploración se pudo constatar su relevancia y la situación de riesgo en que se encontraba una parte del yacimiento, debido a las particulares características de la cueva. Por este motivo se solicitó una autorización para realizar una primera documentación y recogida de los restos humanos, que se llevó a cabo en 2015. A raíz del interés suscitado por los restos recuperados entonces, y por la peculiaridad del yacimiento, iniciamos en 2017 el proyecto de estudio en que se encuadran nuestras actuaciones.

Ostolo es un conducto kárstico activo, de unos ochenta metros de desarrollo, en el que se filtran y acumulan aguas superficiales de las dolinas y depresiones cercanas, y se evacúan a través de una surgencia que constituye el único acceso posible al sistema. La masa de agua forma un pequeño río interior, en cuyos márgenes se han formado dos terrazas de sedimentos y sobre las que se depositaron los restos humanos localizados. 
Estas terrazas están siendo erosionadas por el cauce del río, provocando su desmoronamiento y el arrastre de los restos humanos por la corriente.

El interés del yacimiento radica en la dificultad de acceso a la cueva y en los materiales recuperados hasta el presente. Las evidencias de que disponemos actualmente permiten afirmar que el único acceso practicable al interior de la cueva se realiza a través de la surgencia actual, que es un conducto bajo y estrecho de nueve metros de longitud, por el que solo puede transitarse reptando sobre el cauce del manantial. Una vez en el interior se accede a una sala amplia y de techo alto, donde se sitúa una terraza sobre la que se localizaron los restos.

Estos se agrupan en tres conjuntos separados entre sí: 1) una primera acumulación o Zona 1, con restos desarticulados de al menos seis individuos y donde se recuperó un botón en hueso de doble perforación en $\mathrm{V} ; 2$ ) un segundo conjunto o Zona 2, delimitado por una depresión que alberga unos pocos restos humanos y donde se observa una estructura de combustión (rubefacción del suelo y carbones); y 3) un tercer conjunto o Zona 3, análogo al anterior, pero con un mayor número de restos y donde se localizó un brazal de arquero completo.

Aunque los elementos de ajuar localizados son realmente escasos, el brazal de arquero y el botón de hueso destacan por su alto valor crono-cultural y tipológico, por su rareza en la región y por tratarse de un yacimiento en cueva, ya que este tipo de contextos sepulcrales se ha caracterizado tradicionalmente por la pobreza y poca representatividad de los ajuares.

Nuestra investigación se centra en documentar con el mayor detalle posible las características del yacimiento, que incluyen tanto la documentación y recuperación de los restos arqueológicos propiamente dichos como el estudio de las condiciones del medio físico que lo alberga.

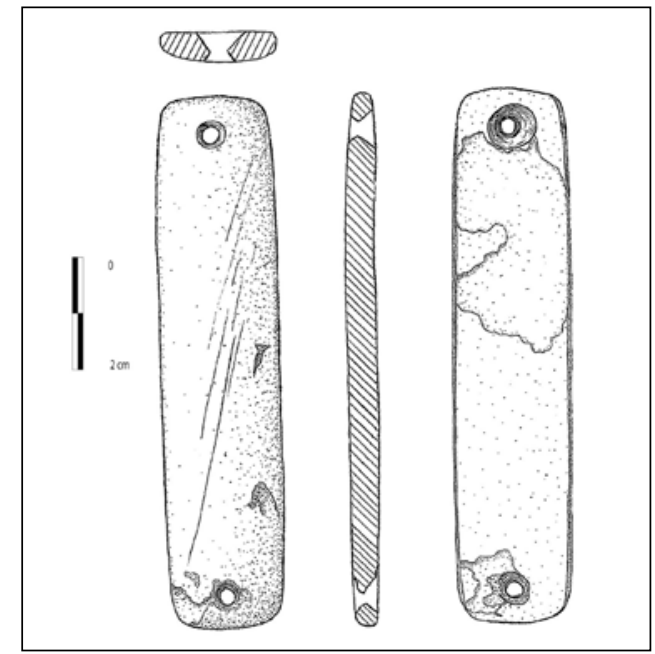

Figura 1. Brazal de arquero hallado en Ostolo (Arantza).

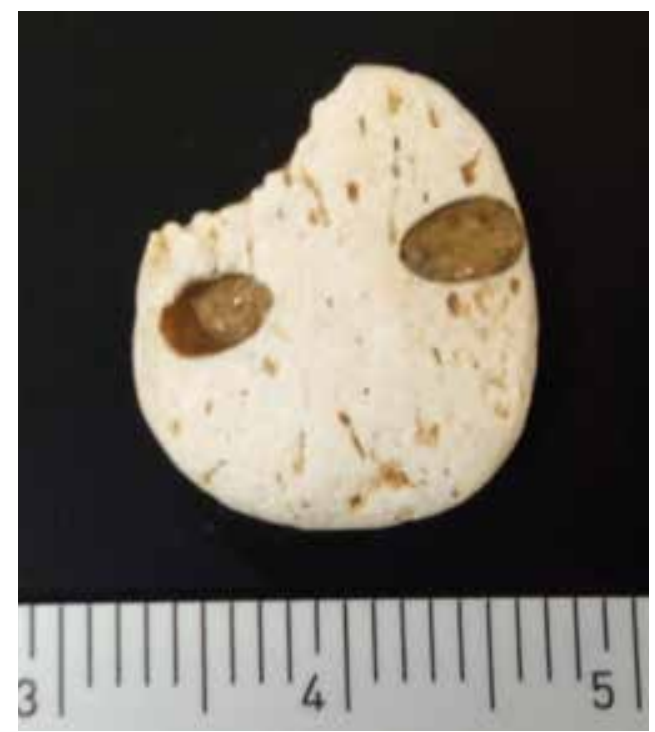

Figura 2. Botón de doble perforación en $\mathrm{V}$, realizado en hueso, hallado en Ostolo. 
Los objetivos arqueológicos son registrar y recuperar con la máxima fidelidad posible los restos en superficie, con el fin de obtener una reconstrucción veraz de cómo se dispusieron los restos y de qué fenómenos han alterado el contexto original. Esto incluye la realización de levantamientos fotogramétricos para la recogida individualizada de cada resto, y ha constituido la tarea de campo principal desde 2017.

Una vez registradas las posiciones de todos los restos, se han puesto en manos de los especialistas que colaboran el proyecto, principalmente para el estudio de los huesos humanos. El objetivo es identificar el número mínimo de individuos, el perfil demográfico de la población representada y una valoración de las partes esqueléticas presentes.

El fin último será determinar si el conjunto es resultado de un proceso acumulativo de mayor o menor duración en el tiempo, y tratar de observar qué tipo de comportamientos determinan la organización espacial interna de los restos.

En cuanto al estudio de las condiciones particulares del medio físico, desde 2018 se desarrolla una línea de investigación paralela con tal objetivo, bajo la dirección de Eneko Iriarte. Nuestro primer esfuerzo fue tratar de esclarecer si las condiciones de acceso pudieron ser distintas a las actuales, más fáciles, durante el uso de Ostolo como lugar funerario en el pasado. En caso de ser similares a las actuales, cabría preguntarse cuál es el sentido de esta elección y qué condicionantes impondría ese hecho para el desarrollo de las prácticas funerarias.

Aunque el estudio de los materiales está aún en marcha, una serie de observaciones preliminares permite plantear unas conclusiones provisionales:

Los muestreos de espeleotemas y la toma de datos realizados desde 2018 muestran un registro continuo de gran longitud temporal y de gran resolución que permitirá una reconstrucción paleoclimática detallada, y sugieren, además, que las condiciones de acceso a la cueva serían análogas a las actuales.

En cuanto a los restos arqueológicos y su distribución, las posiciones de los restos parecen mostrar relaciones de proximidad claras entre elementos anatómicos afines entre sí, e identificar varios individuos. Esto, junto con la presencia de determinadas partes anatómicas (vértebras, costillas, falanges) sugiere que se trata de enterramientos primarios, lo que entraña mayor dificultad aún para ingresar los cuerpos completos en la cueva.

En cuanto al perfil demográfico, las observaciones de campo permitieron identificar individuos de distintos rangos de edad (infantiles, adultos jóvenes y maduros) dentro la Zona 1. Conviene esperar a los resultados del estudio antropológico para establecer si existen perfiles excluyentes entre las tres zonas determinadas. En cuanto a la asociación entre restos humanos y ofrendas/ajuares, tanto en la Zona 1 como en la 3 se han hallado materiales considerados bienes de prestigio propios del complejo campaniforme (botón, brazal). 
Un último elemento inusual es la constatación de que se han alumbrado hogueras en el interior de la cueva, que se han identificado con claridad (por las evidencias de rubefacción y concentración de carbones) en sendas depresiones de las zonas 2 y 3 , y se intuyen también en una repisa sobre la Zona 1.

En cualquier caso, además de la finalización de los estudios en curso, la contrastación de estas hipótesis preliminares pasa por concretar la coherencia cronológica de cada uno de los contextos y del conjunto en su totalidad, lo que requerirá una amplia batería de dataciones.

En esta campaña hemos logrado ya un primer resultado, que sitúa un resto humano de la Zona 3 a inicios de la Edad del Bronce, en fechas coherentes con la aparición de los brazales de arquero y otros elementos del Campaniforme. 